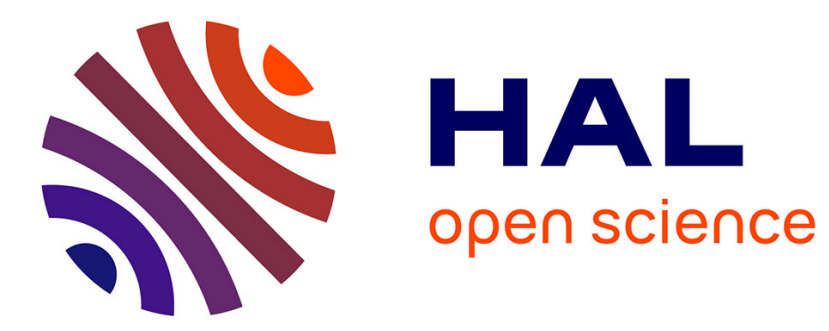

\title{
Composition measurement of bicomponent droplets using laser-induced fluorescence of acetone
}

\author{
C. Maqua, V. Depredurand, G. Castanet, M. Wolff, F. Lemoine
}

\section{To cite this version:}

C. Maqua, V. Depredurand, G. Castanet, M. Wolff, F. Lemoine. Composition measurement of bicomponent droplets using laser-induced fluorescence of acetone. Experiments in Fluids, 2007, 43 (6), pp.979 - 992. 10.1007/s00348-007-0368-1 . hal-01570359

\section{HAL Id: hal-01570359 \\ https://hal.univ-lorraine.fr/hal-01570359}

Submitted on 29 Jul 2017

HAL is a multi-disciplinary open access archive for the deposit and dissemination of scientific research documents, whether they are published or not. The documents may come from teaching and research institutions in France or abroad, or from public or private research centers.
L'archive ouverte pluridisciplinaire HAL, est destinée au dépôt et à la diffusion de documents scientifiques de niveau recherche, publiés ou non, émanant des établissements d'enseignement et de recherche français ou étrangers, des laboratoires publics ou privés. 


\title{
Composition measurement of bicomponent droplets using laser-induced fluorescence of acetone
}

\author{
C. Maqua $\cdot$ V. Depredurand - G. Castanet \\ M. Wolff · F. Lemoine
}

\begin{abstract}
Commercial fuels are complex mixtures, the evaporation of which remains particularly difficult to model. Experimental characterization of the differential vaporization of the components is a problem that is seldom addressed. In this paper, the evaporation of binary droplets made of ethyl-alcohol and acetone is investigated using a technique of measurement of the droplet composition developed in purpose. This technique exploits the laser induced fluorescence of acetone which acts as a fluorescent tracer as well as the more volatile component of the fuel associated with an accurate measurement of the droplet diameter by forward scattering interferometry. A model of the fluorescence intensity of the binary mixture, taking into account the absorption of the acetone molecules, is proposed and validated. The sensitivity of the technique is discussed. Finally, the reliability of the technique is demonstrated on binary combusting droplets in linear stream.
\end{abstract}

\section{List of symbols}

A Spontaneous emission Einstein coefficient

$C=L / D \quad$ Dimensionless distance parameter

$D \quad$ Droplet diameter

$E_{0} \quad$ Incident laser energy

E Laser energy

I Fluorescence intensity

$K_{\text {opt }} \quad$ Optical constant

$k_{\mathrm{q}} \quad$ Quenching constant

$m \quad$ imaginary refractive index

C. Maqua · V. Depredurand - G. Castanet - M. Wolff ·

F. Lemoine $(\bowtie)$

LEMTA, Nancy-Universitè, CNRS, 2, Avenue de la forêt de

Haye, BP 160, 54504 Vandoeuvre-lès-Nancy, France

e-mail: fabrice.lemoine@ensem.inpl-nancy.fr
$\begin{array}{ll}V_{\mathrm{d}} & \text { Droplet volume } \\ t & \text { Time } \\ Z & \text { Volume fraction }\end{array}$

Greek symbols

$\varepsilon \quad$ Molar extinction coefficient

$\chi$ Molar fraction

\section{Subscript}

inj Injection

a Acetone

e Ethanol

1 Local

0 Reference measurement

\section{Introduction}

Commercial fuels are made of hundreds of components which have different volatilities. Pollutant emission, ignition delays and overall combustor efficiency can be directly related to the evaporation of the fuel, thus the multi-component nature of the fuels should be introduced in the predictive tools with the highest accuracy. Quantitative measurements on evaporating multi-component droplets are required to understand spray evaporation of practical fuels and to validate models in combustion devices. Validations should begin on simplified configurations where the different aerothermal parameters, such as droplet size, velocity temperature, spray density, fuel-air ratio can be easily separated. Nonetheless, in an industrial combustor, all the mentioned parameters are generally coupled, which makes it difficult to determine their own influence. As a result, experiments are often performed on idealized sprays in order to minimize the coupling and to provide parametric results. In particular, many studies were performed 
using linear monodisperse droplet streams (Silverman and Dunn-Rankin 1994; Connon et al. 1997; Castanet et al. 2003). Temporally and spatially periodical streams of droplets are generated with well-controlled injection frequency, droplet size, velocity and droplet spacing. This simple geometry is particularly attractive. Since the injection parameters can be adjusted independently, experiments can provide a database for various aerothermal conditions, which is very useful for the validation of computational fluid dynamics codes. In addition, physical models can be derived from such experiments and can be subsequently implemented into simulation codes.

Only few optical diagnoses applied to multicomponent droplets have been reported and most of them are light scattering based techniques. Zhao and Qiu (2004) measured the droplet refractive index by rainbow refractometry in order to determine temperature of binary mixtures of water and ethanol. Wilms et al. (2004, 2006) also used rainbow refractometry to determine the composition of binary droplets of $n$-hexadecane and $n$-dodecane. Since the refractive index depends on both composition and temperature, assumption was made that the temperature of the mixture can be approximated by the equilibrium temperature of pure $n$-hexadecane droplets. Furthermore, the rainbow refractometry technique is highly sensitive to the non-sphericity of the droplets and to refractive index gradients (either due to composition or temperature gradients). The latter is a severe limitation especially in the transient heating phase. On the contrary, LIF-based techniques do not suffer from the same difficulty of separating the influence of the composition and temperature, which make them all the more attractive. Temperature measurements of binary droplets made of acetone and ethyl-alcohol were reported by Maqua et al. (2006) who used the three colors LIF to dissociate the effects of temperature and composition variations.

The fluorescence of acetone induced by UV lasers was widely used for mixture fraction and temperature measurements in the gas phase (Lozano et al. 1992; Thurber et al. 1998). A noticeable extension in two phase flows was reported by Orain et al. (2005), who measured acetone mole fraction distribution in the gas phase surrounding monodisperse droplets streaming linearly. Measurements of vaporized and liquid fuel concentration field in a combusting spray jet of acetone were performed by Bazile and Stepowski (1995), using laser-induced fluorescence of acetone: the strong absorption caused by liquid acetone was pointed leading to a compression of the dynamic of the fluorescence intensity. Then, the fluorescence power from a small drop scales with its volume, whereas it turns out to be proportional to its surface for the big drops. Other quantitative measurements of acetone vapour concentration in presence of droplets were reported by Ritchie and Seitzman
(2001): a model was developed to use PLIF signal to derive droplet size as well by taking into account the disturbing effect of the droplets. Tran et al. (2005) studied particularly the fluorescence and phosphorescence of liquid acetone to assist in the development of quantitative mixing measurements in two-phase flows.

This article relates the development of an instantaneous measurement of the droplet composition in case of a binary mixture of acetone and ethanol; the acetone being the more volatile component. The diagnostic is based on the detection of the laser induced fluorescence signal from acetone in the liquid phase and an accurate measurement of the droplet diameter using forward scattering interferometry. The different stages of the technique development and the fluorescence modelling to derive the acetone fraction are detailed. Finally, to demonstrate the technique, experiments on combusting acetone/ethanol droplets streaming linearly are described and analysed.

\section{Combusting monodisperse droplet stream facilities}

\subsection{The monodisperse droplet stream generator}

A linear monodisperse droplet stream is generated by Rayleigh disintegration of a liquid jet which experiences a mechanical vibration provided by a piezoceramic.

The voltage applied on the piezoceramic is a square wave, the amplitude of which affects the position of the break-up zone. For certain resonant frequencies, the liquid jet breaks up into equally spaced and monosized droplets. The liquid fuel is pressurized with compressed air between 0.2 and 1 bars to force the flow through a calibrated orifice with a diameter ranging from $\Phi_{0}=50 \mu \mathrm{m}$ to $\Phi_{0}=100 \mu \mathrm{m}$. Similar facilities were used in past studies (Lavieille et al. 2001, 2002; Castanet et al. 2005). The droplet injection velocity can range from 2 to about $10 \mathrm{~m} / \mathrm{s}$. The fuel temperature is regulated in the injector body with the help of an external heated water circulation and measured with a K-type thermocouple. A laminar spray flame is ignited by means of an electrically heated coil, placed just after the break-up zone of the liquid jet (Fig. 1). In such a configuration of stream, the dimensionless spacing parameter $C$ is defined as the ratio between the droplet spacing $L$ and the droplet diameter $D$ :

$C=L / D$

\subsection{Droplet diameter and velocity measurements}

Knowledge of the droplet size reduction is necessary to characterize the evaporation of the droplets. The 


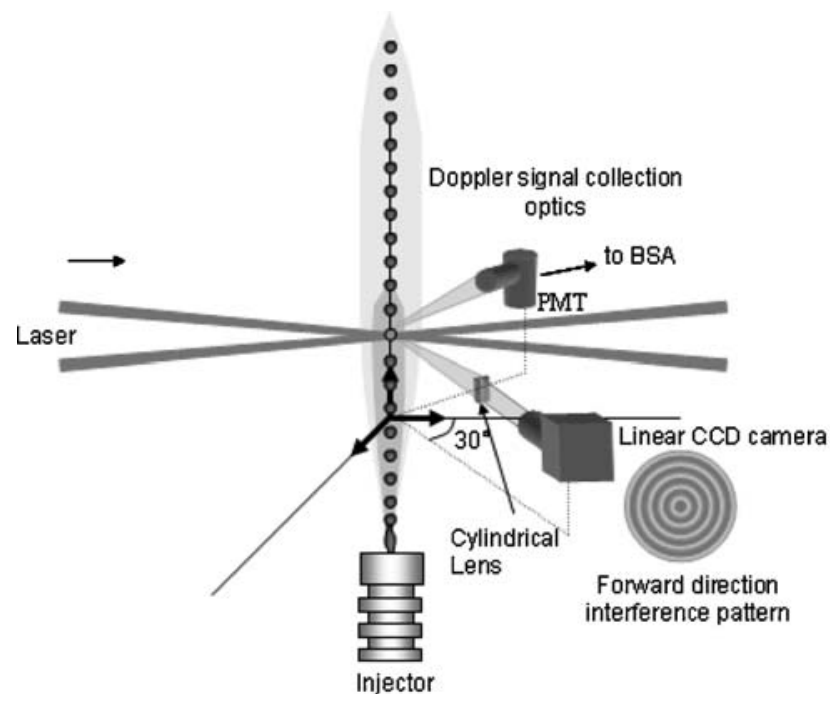

Fig. 1 Sketch of a combusting monodisperse droplet stream with the droplet diameter measurement facilities

measurement technique is based on the interaction between a spherical droplet and a laser beam (Koenig et al. 1986). A beam issuing from an argon ion laser is focused on a droplet (Fig. 1). Interferences between reflected rays and one time refracted rays are generated in the forward direction. The high frequency of the droplets passage makes that the fringe pattern appears stationary. Measurement of the angular interfringe near the forward scattering angle of $30^{\circ}$ enables to determine the droplet diameter within $\pm 0.5 \mu \mathrm{m}$ with a very limited sensitivity to the droplet refractive index (Massoli 1998). The interference pattern is focused in one direction by means of a cylindrical lens in order to increase the light power density. Image of the spatial intensity distribution is formed on the linear sensor chipset of a CCD camera (4,096 pixels). The angular interfringe is measured between two interference maxima in order to derive the droplet size as suggested by Frohn and Roth (1998). The droplet velocity is measured simultaneously by an additional LDA system. With the help of the droplet velocity, distance from the injector exit can be converted easily into time.

\section{Fluorescence of liquid acetone}

\subsection{General concepts}

In the present experiment, acetone acts as an evaporating fuel as well as a fluorescent tracer for the liquid phase. In a bicomponent mixture, one of the components (acetone in the present case) can be excited to fluoresce. The total emitted fluorescence can be used as a measurement of the number of molecules of the fluorescing component. In a monodisperse droplet stream and with the use of an independent and accurate measurement of the droplet diameter, this information can be used to derive the relative concentration of the fluorescing component in the droplet.

Acetone was chosen as a fluorescent tracer for two main reasons:

1. Acetone can represent as a well volatile component of a fuel and presents interesting fluorescence properties that can be used for measurements.

2. Fluorescence of the vapor of acetone in the vicinity of the droplets can be used in the future to measure the vapor distribution of the volatile component in the gas phase.

However, the drawback of using acetone is related to the strong absorption of this molecule in the liquid phase, which makes the measurement strategy more complicated and requires corrections.

The absorption of liquid acetone is very efficient at $266 \mathrm{~nm}$ which corresponds to the radiation of a quadrupled Nd-Yag laser (Fig. 2). The resulting fluorescence spectrum is broadband with a maximum emission in the $420-520 \mathrm{~nm}$ region (Fig. 2). It was decided to detect the fluorescence on a narrow spectral band centered at $450 \mathrm{~nm}$ (bandwidth $\Delta \lambda=20 \mathrm{~nm}$ ) to avoid any residual radiation of the second harmonic of the Nd-Yag laser at $532 \mathrm{~nm}$.

LIF results from a process that begins with the absorption of the incident light by the acetone molecules which are promoted to a higher energy level. Some of the excited molecules return to the ground state by emitting fluorescence. The intensity of the fluorescence signal, collected

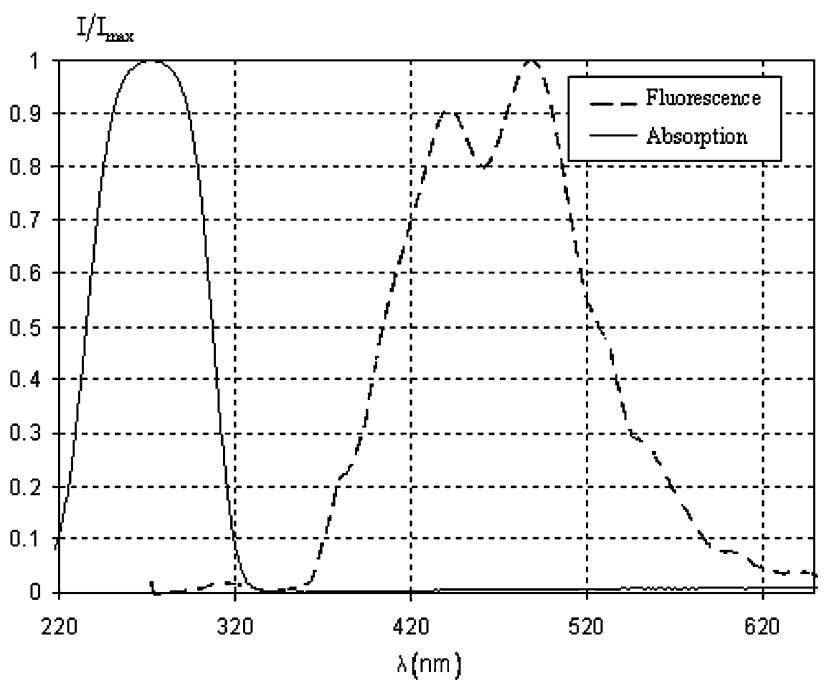

Fig. 2 Normalized absorption and fluorescence spectra of liquid acetone (fluorescence is induced by a frequency quadrupled Nd-Yag laser) 
from an elementary volume $\mathrm{d} V$ assuming no saturation, is given by Lemoine et al. (1999):

$\mathrm{d} I=K_{\text {opt }} E_{\mathrm{l}} \mathrm{d} V C_{\mathrm{a}} \varepsilon_{\mathrm{a}} \eta$

where $K_{\text {opt }}$ is a constant specific to the optical device, $E_{1}$ is the local incident laser energy per pulse per unit area, $C_{\mathrm{a}}$ is the molecular concentration of the absorbers, i.e., acetone molecules. $\varepsilon_{\mathrm{a}}\left(\mathrm{mol}^{-1} \cdot 1 . \mathrm{m}^{-1}\right)$ is the molar absorption coefficient for the acetone molecule at $266 \mathrm{~nm}$ and $\eta$ is the fluorescence quantum yield of acetone and takes into account all non-radiative decay processes including collisionnal quenching. The optical constant $K_{\text {opt }}$ can then be evaluated using a suitable reference. It depends on experimental parameters such as probe volume, solid angle, spectral and spatial efficiencies of filters and detection devices. The local intensity $E_{1}$ can be related to the laser incident intensity $E_{0}$ by the Beer's absorption law, if the absorbing specie concentration $C_{\mathrm{a}}$ is uniform and the beam trajectory is rectilinear, by:

$E=E_{0} e^{-\varepsilon_{\mathrm{a}} C_{\mathrm{a}} x}$

where $x$ is the optical path of the incident laser beam.

The molar extinction coefficient of acetone at $266 \mathrm{~nm}$ was measured by absorption spectroscopy. The measurement is performed for a very low volume fraction of acetone dissolved in ethyl-alcohol which does not absorb at $266 \mathrm{~nm}$. The value inferred from these experiments is $\varepsilon_{\mathrm{a}}=3,552 \pm 106 \mathrm{~mol}^{-1} \cdot 1 \cdot \mathrm{m}^{-1}$ and is quite insensitive to the fraction of ethanol in the solution. To compare with previous calculations detailed in the literature, the imaginary part of the refractive index of pure acetone at $266 \mathrm{~nm}$ is on the order of $m=10^{-3}$. Consequently, the optical thickness of liquid acetone corresponding to an extinction of $99 \%$, is about $95 \mu \mathrm{m}$, which is comparable to the order of magnitude of the droplet sizes considered in this study.

The fluorescence quantum yield $\eta$ should depend on liquid phase composition (i.e., acetone fraction and temperature). However, it will be shown that their variation is almost negligible in the temperature range of the liquid phase.

The general expression of the fluorescence quantum yield is:

$\eta=A /\left(A+Q_{1}+Q_{2}\right)$

where $Q_{1}$ is the collisional quenching rate, $Q_{2}$ is the rate of non-radiative decay by any other processes than collisions. In the case of acetone, $Q_{2}$ corresponds mainly to intersystem crossing (Thurber et al. 1998). $A$ is the Einstein coefficient for spontaneous emission.

The collisional quenching rate $Q_{1}$, for a given molecular specie can be expressed by (Glowacki 1964):
$Q_{1}=k_{\mathrm{q}} C_{\mathrm{M}}$

where $k_{\mathrm{q}}$ is the kinetic quenching constant and $C_{\mathrm{M}}$ is the molar concentration of the collision partners. In the case of an acetone-ethanol mixture, acetone fluorescence can by quenched by both ethanol and acetone molecules. The collisional quenching rate depends on the liquid composition:

$Q_{1}=k_{\mathrm{q} 1} C_{\mathrm{a}}+k_{\mathrm{q} 2} C_{\mathrm{e}}$

where $k_{\mathrm{q} 1}$ is the kinetic constant related to the quenching of acetone fluorescence by the acetone molecules (selfquenching) and $k_{\mathrm{q} 2}$ to the quenching of acetone fluorescence by the ethanol molecules. The quenching constants are likely to depend on temperature (Glowacki 1964; Lemoine et al. 1999).

Since $Q_{2}$ is unknown, a simple empirical correction of the fluorescence quantum yield, to account for the dependence of this parameter on the liquid composition, was introduced.

By introducing the molar fraction of acetone $\chi_{\mathrm{a}}$, the fluorescence signal will be expressed by:

$\mathrm{d} I=K_{\text {opt }} K_{\text {spec }} E_{\mathrm{l}} \mathrm{d} V \chi_{\mathrm{a}} \frac{1}{\chi_{\mathrm{a}} \mu+1}$

where $K_{\text {spec }}$ is a spectroscopic which includes Einstein coefficient for spontaneous emission and molar absorption coefficient and $\mu$ is a dimensionless empirical constants, which will be properly calibrated in the experimental procedure.

\subsection{Fluorescence in a droplet}

\subsubsection{Governing equation}

In this section, the case of a droplet completely illuminated by a plane wave will be examined. The probe volume $V$ is therefore replaced by the droplet volume $V_{\mathrm{d}}$ in Eq. (7). To evaluate the local excitation energy $E_{1}$ in the droplet, the focusing effect of the spherical interface and the noticeable absorption must be taken into account. Previous investigations, carried out by Le Gal et al. (1999), show that in the case of strong absorption, the droplet volume is only partially illuminated by the laser radiation resulting in a fluorescence signal not directly proportional to the droplet volume. The absorption, depending on the droplet diameter and acetone fraction, tends to decrease the efficiency of the excitation by the incident laser beam. Thus, the efficiency of the laser excitation can be defined, according to the following expression: 
$\xi\left(\chi_{\mathrm{a}}, D\right)=\frac{\int_{V_{\mathrm{d}}} E\left(x, y, z, \chi_{\mathrm{a}}, D\right)}{\int_{V_{\mathrm{d}}} E\left(x, y, z, \chi_{\mathrm{a}}=0, D\right)}$

where $E\left(x, y, z, \chi_{\mathrm{a}}, D\right)$ is the internal energy field at given acetone fraction $\chi_{\mathrm{a}}$ and droplet diameter $D$ and $E(x, y, z$, $\left.\chi_{\mathrm{a}}=0, D\right)$ is the same quantity assuming no absorption. The internal field can be calculated by a ray tracing method using the 3D Snell's law at the droplet interface and taking into account the absorption along the ray path according to Eq. (3). Only first order rays refracted at the droplet surface are considered, according to the Fresnel coefficient. Higher order rays resulting from one or more internal reflections are neglected, because of the strong absorption generated by acetone. To assess the reliability of this approach, a calculation of $\xi\left(\chi_{\mathrm{a}}, D\right)$ was carried out for one test case corresponding to $D=80 \mu \mathrm{m}$ using the Generalized Lorentz-Mie theory (GLMT) developed by Gouesbet and Grehan (2000). As seen in Fig. 3, the two approaches are in very good agreement.

After collection all over the droplet volume, the fluorescence signal is expressed by:

$I=K_{\text {opt }} K_{\text {spec }} \frac{\chi_{\mathrm{a}}}{\chi_{\mathrm{a}} \mu+1} V_{\mathrm{d}} E_{0} \xi\left(\chi_{\mathrm{a}}, D\right)$

From Eq. (9), it can be seen that the determination of the acetone fraction $\chi_{\mathrm{a}}$ implies the kowledge of the correction factor $\mu$ for the quenching term, as well as an accurate measurement of the droplet size $V_{\mathrm{d}}$. A preliminary calibration of the quenching parameter $\mu$ will be presented in Section 3.3.

\subsubsection{Temperature dependence}

The molar absorption coefficient $\varepsilon_{\mathrm{a}}$ and the quenching constants $k_{\mathrm{q} 1}, k_{\mathrm{q} 2}$ and $Q_{2}$ are likely to be temperature

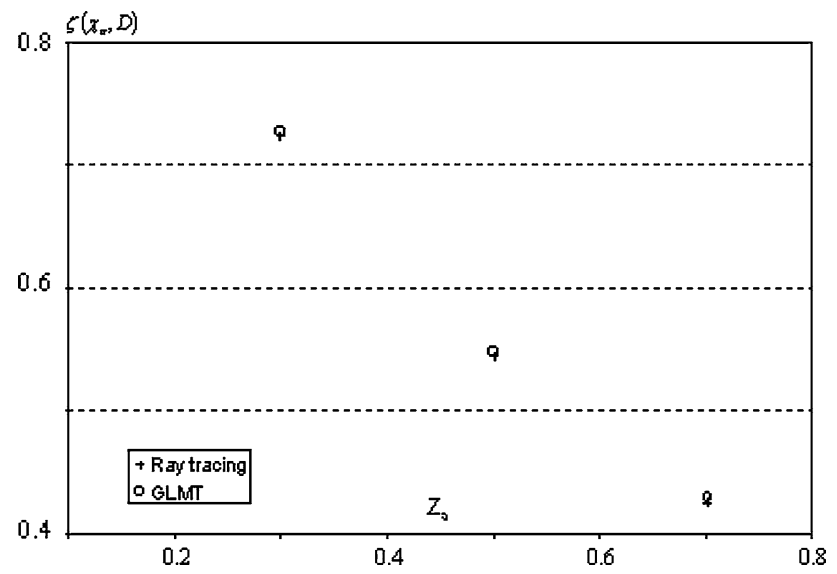

Fig. 3 Comparison of the efficiency factor calculated by ray tracing and $\operatorname{GLMT}(D=80 \mu \mathrm{m})$ dependant. Even though most articles dealing on the topic concern gaseous acetone (Thurber et al. 1998), the effect of temperature on the absorption cross section and the overall fluorescence signal is expected to be negligible at an excitation wavelength of $266 \mathrm{~nm}$, since the investigated range of temperature is generally limited by the boiling point of the more volatile component (acetone, $56^{\circ} \mathrm{C}$ ) in such a situation.

The liquid density changes with the temperature which may also have an influence on the fluorescence intensity in the conversion of volume fractions to molar fraction and also in the efficiency factor $\xi\left(\chi_{\mathrm{a}}, D\right)$, where the knowledge of the acetone volume concentration is essential. All the calculations will be performed assuming constant and uniform droplet temperature. However, the deviation caused by this assumption will be evaluated.

\subsection{Preliminary studies}

\subsubsection{Initial calibrations}

The parameter $\mu$ in Eq. (9) is a priori unknown parameter and must be determined by a calibration. This calibration is performed on a liquid cylindrical jet of ethanol-acetone mixtures. This kind of setup was preferred, since it avoids photobleaching and limits the optical path due to the high absorption of acetone in contrast to a cell. The layout is depicted in Fig. 4: the liquid jet is generated by a syringe with a thin needle. The needle length was selected to avoid any instabilities and oscillations of the outcoming liquid jet in the measurement zone, which was controlled by visualization using a high magnification. The laser source is a quadrupled Nd-Yag, delivering radiations at $266 \mathrm{~nm}$, with pulse duration of $7 \mathrm{~ns}$ and a repetition rate of $10 \mathrm{~Hz}$. The

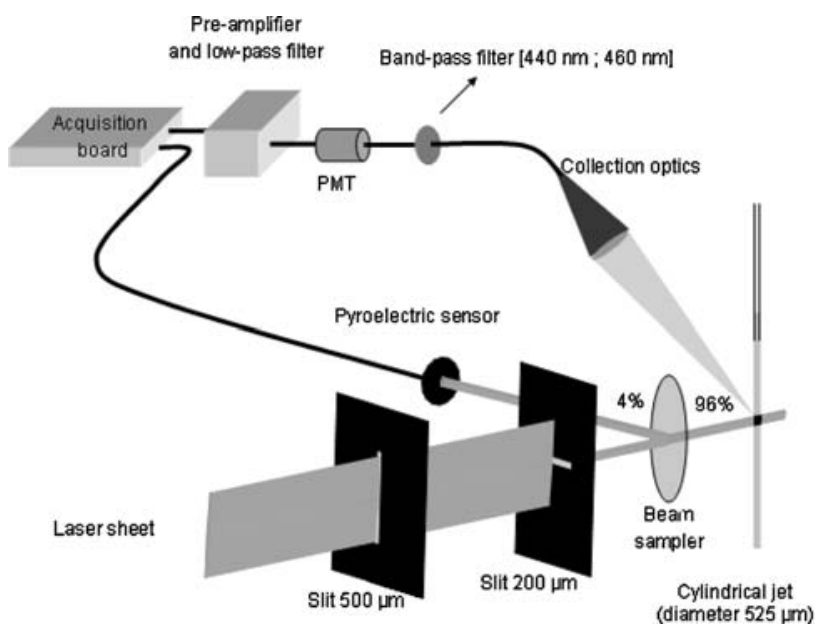

Fig. 4 Block-diagram of the calibration experiment that uses a cylindrical jet 
laser beam (diameter $9 \mathrm{~mm}$ at $1 / e^{2}$ ) is focussed along one direction by means of a cylindrical lens and spatially filtered by two perpendicular slits $(200 \times 500 \mu \mathrm{m})$ to reduce the excitation volume before impinging the liquid jet. The incident energy is measured by a pyroelectric detector on the laser beam path positioned just behind the liquid jet. The emitted fluorescence is collected at right angle by an achromatic doublet focusing a $500 \mu \mathrm{m}$ sized spot, before passing through the interference filter and being detected by a photomultiplicator tube. The electric signal is lowpass filtered and amplified before being processed by an acquisition board. The fluorescence intensity was measured for five acetone volume fractions and for increasing laser energies. The results are summed up in Fig. 5 for $Z_{\mathrm{a}}=10$, 60 and $100 \%\left(Z_{\mathrm{a}}=20\right.$ and $80 \%$ are not represented to improve readability). As expected, the fluorescence intensity increases linearly with the laser energy. For the highest incident energies, saturation appears followed by a decrease of the fluorescence intensity. This latter effect can be potentially attributed to pre-dissociation phenomena (Majumder et al. 1999). The slope $\gamma$ in the linear region (smallest acetone fractions) is the highest for the smallest acetone fractions which may be explained considering that:

- the strong absorption by acetone, which increases with increasing acetone concentration, and

- the acetone molecules have a greater quenching efficiency than ethanol. This effect is included in the quenching parameter $\mu$.

To take into account the absorption effect, an efficiency factor $\xi\left(\chi_{\mathrm{a}}, D_{\text {cyl }}\right)$ as defined in Eq. (8), was calculated by the ray tracing code adapted to the geometry of the cylindrical jet. To find the parameter $\mu$, the slope $\gamma$ must be

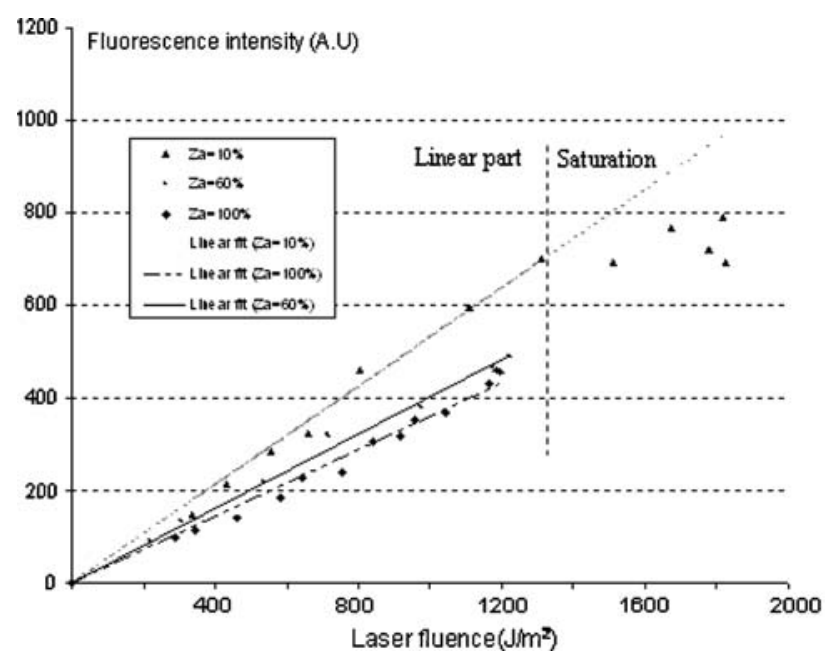

Fig. 5 Evolution of the fluorescence intensity as a function of the incident laser fluence for different acetone fractions in the liquid jet divided by the efficiency factor. The corrected slope $\gamma_{\text {cor }}$ is given by:

$\gamma_{\text {cor }}=\frac{\gamma}{\xi\left(\chi_{\mathrm{a}}, D_{\mathrm{cyl}}\right)}=K_{\mathrm{opt}} K_{\mathrm{spec}} \frac{\chi_{\mathrm{a}}}{\chi_{\mathrm{a}} \mu+1} V$

where $D_{\text {cyl }}$ is the cylinder diameter and $V$ is probe volume.

The evolutions of the corrected slopes $\gamma_{\text {cor }}$ and of the efficiency factors $\xi\left(\chi_{\mathrm{a}}, D\right)$ are presented in Fig. 6 as a function of the acetone fraction. As expected, taking into account the previous corrections, the highest acetone fractions correspond to the highest fluorescence intensity. The calculation of the ratio between the corrected slopes of two molar fractions $\chi_{a i}$ and $\chi_{a j}$, enables to eliminate the optical and spectroscopic constants in Eq. (10). Then, $\mu$ can be derived according to the ratio:

$\gamma_{i \mathrm{cor}} / \gamma_{\mathrm{jcor}}=\left(\frac{\chi_{a i}}{\mu \chi_{a i}+1}\right) /\left(\frac{\chi_{a j}}{\mu \chi_{a j}+1}\right)$

The evaluation of $\mu$ can be repeated using different couples of acetone fractions, which improves ultimately the accuracy after averaging. $\mu$ is found equal to $1.15 \pm 0.09$. The good agreement between the experimental data and the model (with $\mu=1.15$ ) can be checked in Fig. 6.

\subsubsection{Fluorescence signal evaluation}

The fluorescence intensity given by Eq. (9), was computed in order to evaluate its sensitivity to a variation of the acetone volume fraction. The predicted evolution of the fluorescence intensity as a function of the acetone volume fraction is presented in Fig. 7 for different droplet diameters. As expected, the fluorescence intensity exhibits a pronounced dependence on the droplet diameter. Additionally, for a given droplet diameter, the variation of the fluorescence intensity with the acetone fraction is very steep for the lowest acetone fractions. A quasi-linear region can be pointed out with an upper limit at $Z_{\mathrm{a}}=10 \%$. The sensitivity to acetone fraction begins to decrease after $Z_{\mathrm{a}}=10 \%$, but remains noticeable up to $Z_{\mathrm{a}}=30 \%$. For acetone fractions higher than $30 \%$, the sensitivity is very limited. This can be attributed to the balance between the increase of the acetone fraction and the resulting extinction of the incident laser beam by absorption, which reduces the fraction of the droplet volume that contributes to the fluorescence signal. The decrease of the fluorescence intensity for the acetone fractions higher than $Z_{\mathrm{a}}=30 \%$ can be explained by the correction of the quenching term with the fuel composition. This correction tends to decrease the fluorescence efficiency for increasing acetone fractions. The evolution of the efficiency factor $\xi\left(\chi_{\mathrm{a}}, D\right)$, computed by the 
Fig. 6 Evolution of the parameter $\gamma_{\text {cor }}$ (measurements and model) and the efficiency factor in the case of the cylindrical jet, as a function of the acetone fraction

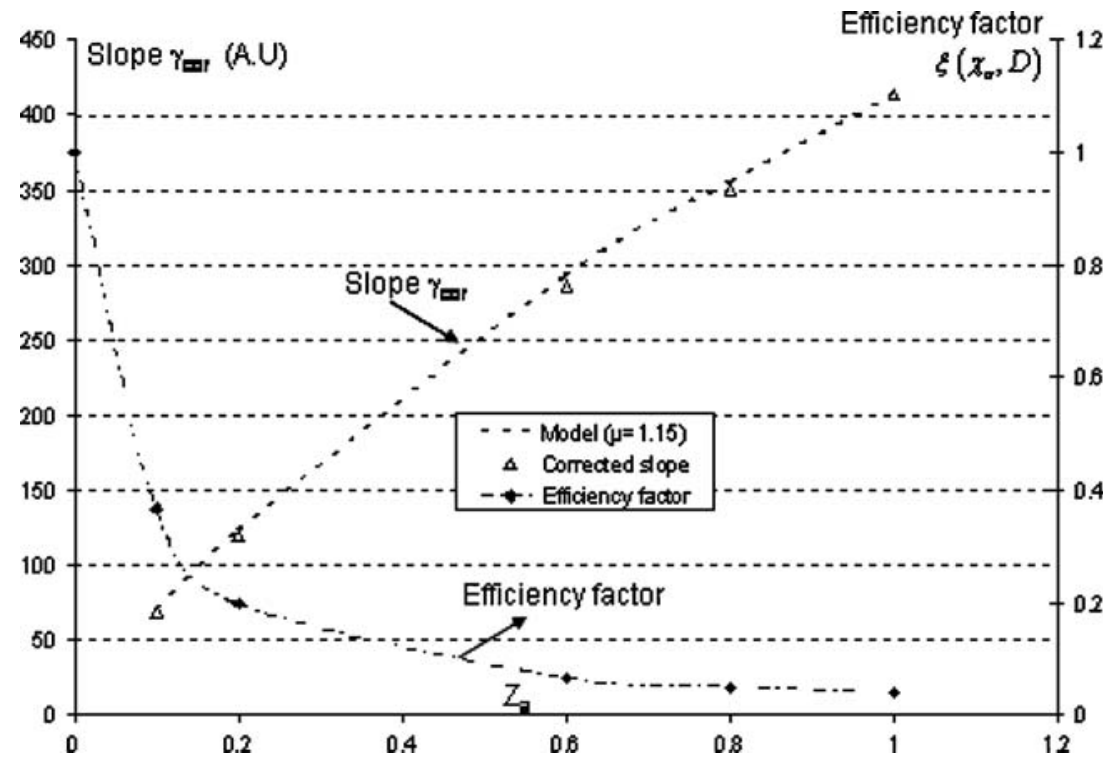

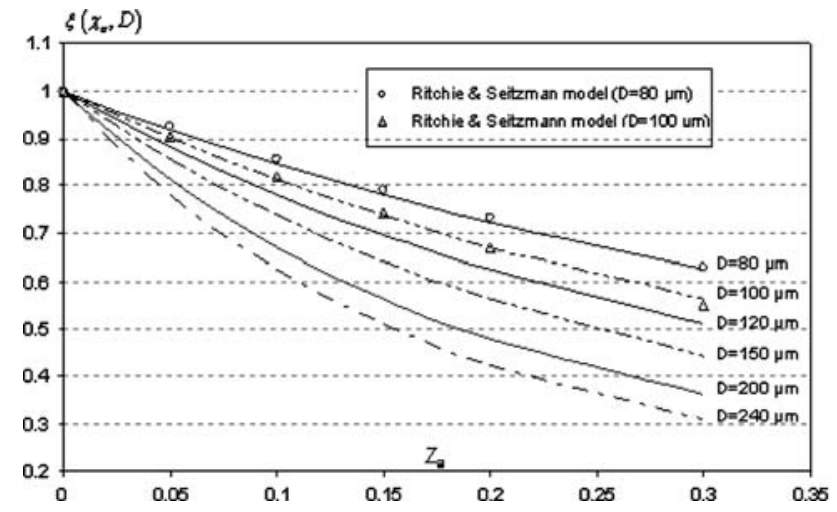

Fig. 8 Efficiency factor as a function of the acetone volume fraction and for different droplet diameters. Comparison to Ritchie and Seitzman model for $D<100 \mu \mathrm{m}$

fluorescence is acquired on the array detector of an electron multiplier CCD camera, equipped with a high magnification objective. The fluorescence signal detected by the camera is digitalized on 14 bits, i.e., 16,384 grey levels. The laser shot is triggered on the piezoceramic frequency at which the droplets are generated. A phase shifter allows adapting the trigger frequency to take into account the time delay due to the displacement of the droplets from the injector exit to the measurement position. The camera acquisition is triggered on the laser pulse. The camera is equipped with the already used band pass interference filter to isolate a spectral band of the fluorescence signal (Section 3.1). A sample of the laser beam is deviated in order to measure the energy per pulse by means of a pyroelectric detector. The energy measurement is done synchronously with the image acquisition by the CCD camera, in order to normalize the fluorescence intensity by the incident energy and to account for the pulse to pulse variation of the laser energy (Fig. 9).
In the present setup, the full aperture of the laser beam $(9 \mathrm{~mm}$ at $\left.1 / e^{2}\right)$ is used to induce the fluorescence of the droplets. The

\section{Measurements on droplets}

\subsection{Experimental setup and processing}




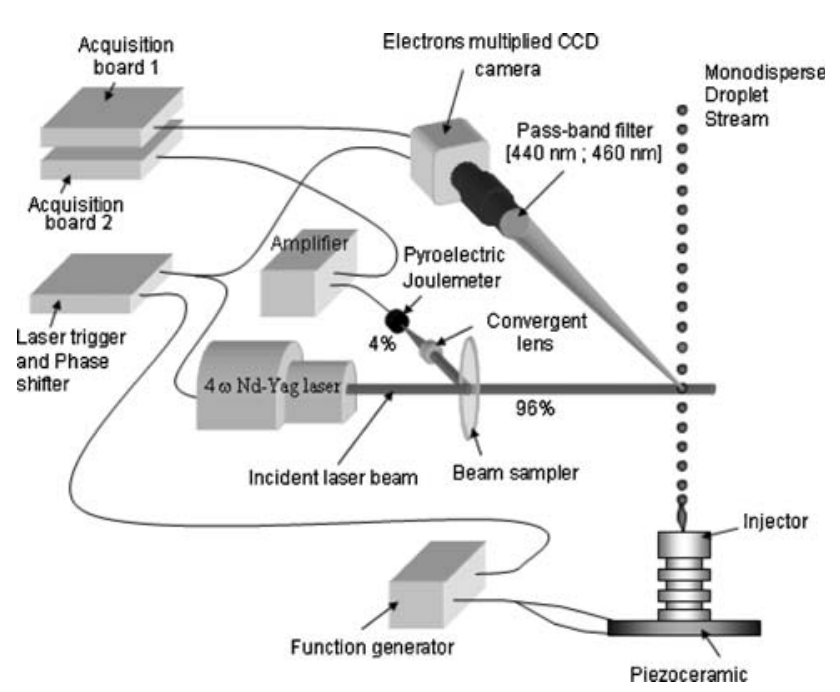

Fig. 9 Block diagram of the measurement layout

The images can be processed differently depending on the targeted objective. The first processing aims to obtain an image of the fluorescence intensity within the droplet. The contour of the droplet is then extracted using a threshold fixed at 1.5 time the background noise and 200 images are averaged after repositioning of the droplet. A background noise image recorded preliminarily is subtracted. A test of this method was performed for two droplets with a diameter $D=109 \mu \mathrm{m}$ and $D=187 \mu \mathrm{m}$ and an acetone fractions $Z_{\mathrm{a}}=20 \%$ and $Z_{\mathrm{a}}=90 \%$, respectively (Fig. 10). The experimental images were compared to the results of the numerical simulation with the $3 \mathrm{D}$ ray tracing code.

To this end, slices of the 3D intensity field calculated by ray tracing cannot be used directly, since they have to be corrected to account for the distortion caused by the droplet spherical interface. The same procedure based on geometrical optics is then used; a wide set of rays is launched under an observation angle at $90^{\circ}$ and the intensity is integrated over each ray path to reconstruct the image. Due to the small dimensions of the droplets, it can be assumed that the intensity is integrated over the whole depth of field of the camera. A good agreement between the experimental and calculated intensity maps can be noticed. The influence of tunnelling waves near the droplet surface cannot be observed, which differs from the observations of Domann and Hardalupas (2001) and Domann et al. (2002). This may be attributed to differences in the value of the imaginary part of the refractive index, varying from $2.10^{-4}$ at $\chi_{\mathrm{a}}=20 \%$ and $9.10^{-4}$ at $\chi_{\mathrm{a}}=90 \%$ in the present work while it ranges from $10^{-5}$ to $5.10^{-4}$ in the previous work of Domann and Hardalupas.

On the experimental images of Fig. 10, no fluorescence coming from the acetone vapour can be seen. This can be explained by the considerable density difference between the liquid and gas phase, which is about 330 . Consequently,
Fig. 10 Excitation field within the droplet (left: calculation by ray tracing; right: experiment)
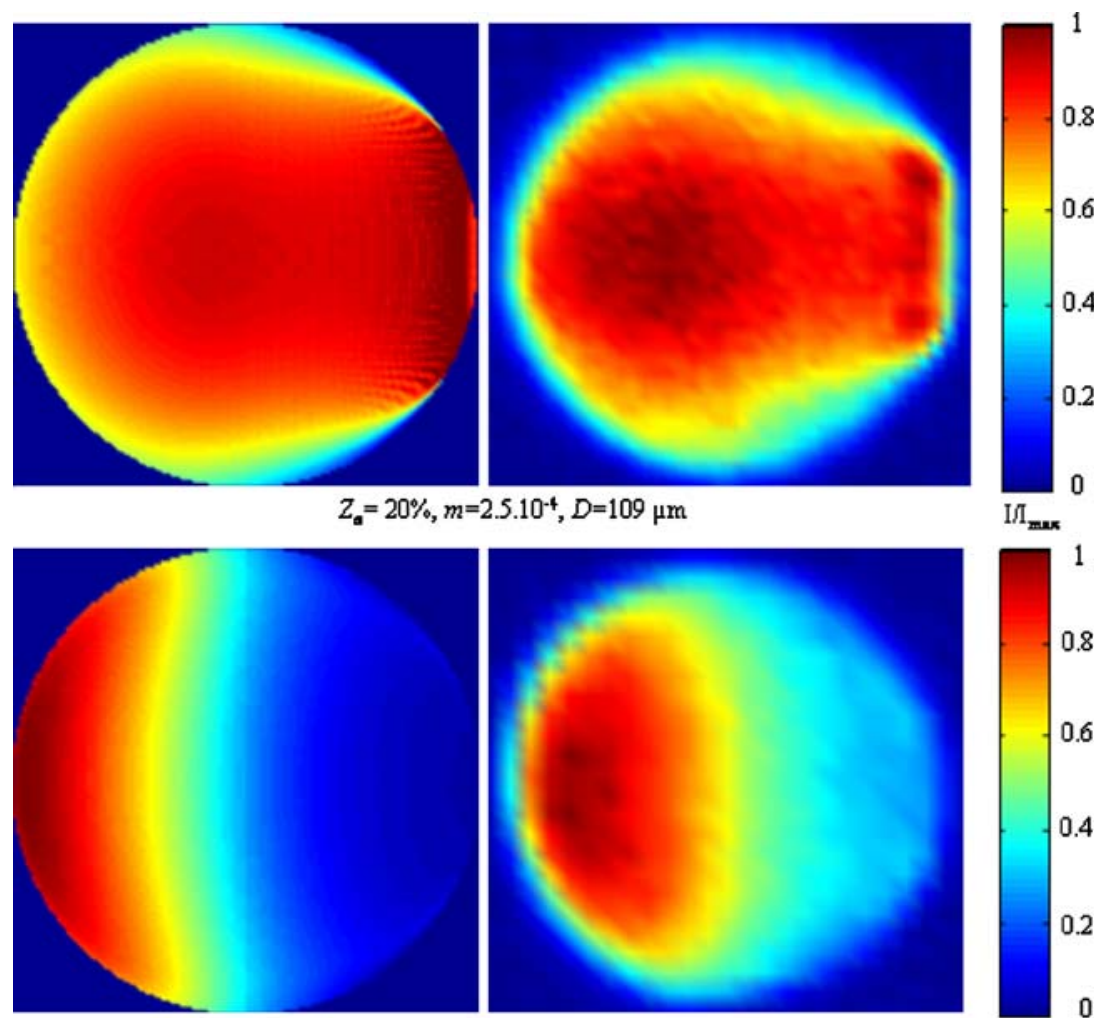

$Z_{\mathrm{a}}=90 \%, m=9 \cdot 3 \cdot 10^{-4}, D=187 \mu \mathrm{m}$

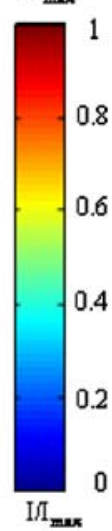


the fluorescence of the gaseous phase was far under the background noise.

When the spaced-averaged composition of the droplets is wished, a region of interest is defined within the images and includes this time several droplets. The images resulting of 200 laser shots are normalized by the energy of each laser pulse and after subtraction of the background noise the signal is averaged over all the images and over the region of interest.

\subsection{Calibrations on droplets}

\subsubsection{Influence of the droplet diameter}

Several droplets sizes were generated by tuning the frequency of the piezoceramic of the monodisperse jet. Data were collected for different droplet diameters (from 80 to $132 \mu \mathrm{m})$ and for two acetone fractions $\left(Z_{\mathrm{a}}=10 \%\right.$ and $\left.Z_{\mathrm{a}}=20 \%\right)$. The measurements were performed after formation of the spherical droplets, but sufficiently close to the injector exit, to neglect evaporation and subsequent variations in the acetone fraction. Furthermore, as the droplet trajectories are very stable close to the injector exit, the droplets are exposed to the same profile of energy when they cross the laser beam. The evolution of the fluorescence intensity as a function of the droplet diameter is presented in Fig. 11 for the two acetone fractions. To avoid non-spherical droplets, the maximum size was limited to $D=135 \mu \mathrm{m}$. As expected the fluorescence intensity increases with the droplet diameter and with the acetone volume fraction. Here also, a good agreement with the model of Eq. (9) is observed in the investigated size range.

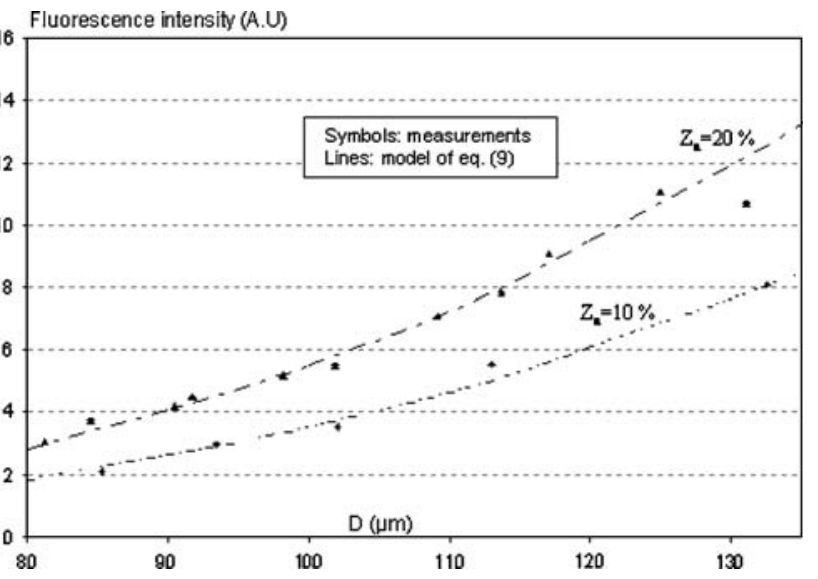

Fig. 11 Measurements of the fluorescence intensity as a function of the droplet diameter for $Z_{a}=10 \%$ and $Z_{a}=20 \%$. Comparison to the model

\subsubsection{Influence of the acetone fraction}

The influence of the acetone fraction was also tested for three droplet diameters $(D=90 \mu \mathrm{m}, D=150 \mu \mathrm{m}$ and $D=200 \mu \mathrm{m})$ and three acetone fractions $\left(Z_{\mathrm{a}}=5 \%\right.$, $Z_{\mathrm{a}}=10 \%$ and $Z_{\mathrm{a}}=20 \%$ (Fig. 12). The evolution of the fluorescence intensity is presented as a function of the acetone fraction and for the three droplet diameters. Model given by Eq. (9) seems to follow the experimental evolution with a rather good accuracy in the investigated range of acetone fraction. Nevertheless, the discrepancy of measurements with the model seems more pronounced for the biggest droplets, which may be due to possible nonspherical droplets.

\subsubsection{Temperature dependence}

In Fig. 12, theoretical values from Eq. (9) were estimated assuming the liquid to be at ambient temperature $\left(T=20^{\circ} \mathrm{C}\right)$ for the conversion of the volume fraction $Z_{\mathrm{a}}$ into molar fraction $\chi_{\mathrm{a}}$ and for the calculation of the efficiency factor $\zeta\left(\chi_{\mathrm{a}}, D\right)$. Since the specific density of acetone evolves from $749 \mathrm{~kg} / \mathrm{m}^{3}$ at $20^{\circ} \mathrm{C}$ to $758 \mathrm{~kg} / \mathrm{m}^{3}$ at $56^{\circ} \mathrm{C}$ while the one of ethanol varies from 782 to $786 \mathrm{~kg} / \mathrm{m}^{3}$ in the same temperature range, the resulting discrepancy is expected to be rather moderate. An additional evaluation of the fluorescence intensity was performed at the maximum expected temperature, i.e., the boiling temperature of acetone $\left(T=56^{\circ} \mathrm{C}\right)$. The deviation with the model at $20^{\circ} \mathrm{C}$ remains negligible for acetone volume fractions less than $Z_{\mathrm{a}} \leq 30 \%$.

To derive the fluorescence model of Eq. (9), it was also assumed that the temperature dependence of the absorption cross section and quenching constants was negligible in the

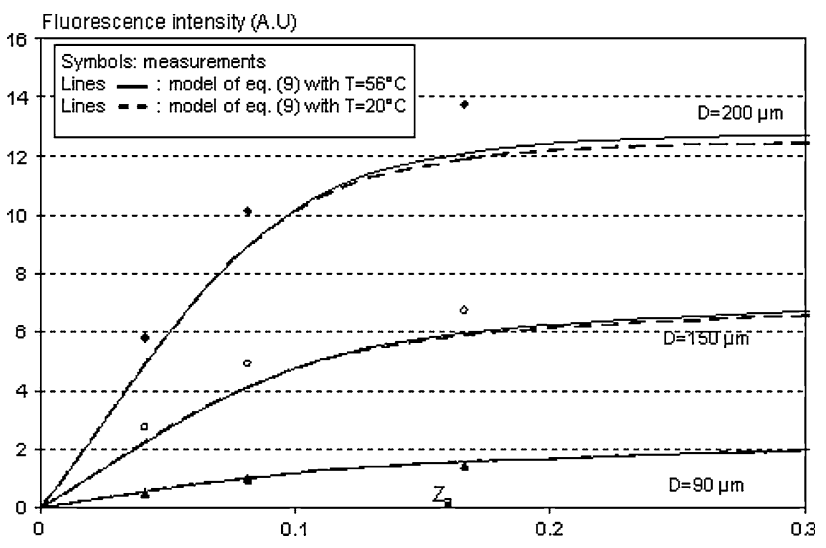

Fig. 12 Measurements of the fluorescence intensity as a function of the acetone fraction for three droplet diameters. Comparison to the model for $T=20^{\circ} \mathrm{C}$ and $T=56^{\circ} \mathrm{C}$ 
experimental conditions, where the temperature of the liquid phase in not likely to exceed the boiling temperature of acetone in the present study $\left(56^{\circ} \mathrm{C}\right)$. This assumption was checked by measuring the fluorescence signal for three temperatures $\left(25,35\right.$ and $\left.45^{\circ} \mathrm{C}\right)$ for $Z_{\mathrm{a}}=20 \%$ in a temperature controlled and agitated cell. The measured variations where randomly scattered within $\pm 0.25 \%$ of the mean value of the fluorescence intensity (Fig. 13).

\section{Experimental results}

\subsection{Measurement process}

In measurement conditions, the trajectories of the droplets in the laser beam may be not perfectly steady due to some turbulence that can be generated by the flame. This affects the fluorescence signal since the distribution of the energy in the laser beam is not homogeneous. In order to reduce the statistical bias induced by these unwanted effects, it is preferred to do the averaging of the fluorescence signal over a region of interest containing four droplets, i.e, $1.5 \mathrm{~mm}$ in high (Fig. 14).

In combustion experiments, the reference measurement is previously taken close to the nozzle exit, when the droplets are formed, without any flame. The reference diameter $D_{0}$ is determined by using the measured injected flowrate $Q$ and the piezoceramic frequency $f\left(D_{0}=\right.$ $\left.(6 Q / \pi f)^{1 / 3}\right)$. Since the composition is supposed to be well known at this point (i.e., equal to the initial composition), the optical and spectroscopic constants can be eliminated and the acetone fraction is then derived from:

$$
\frac{I^{*}}{I_{0}^{*}}=\left(\frac{\chi_{\mathrm{a}}}{\chi_{\mathrm{a}} \mu+1}\right) V_{\mathrm{d}} \xi\left(\chi_{\mathrm{a}}, D\right) /\left(\frac{\chi_{\mathrm{a} 0}}{\chi_{\mathrm{a} 0} \mu+1}\right) V_{\mathrm{d} 0} \xi\left(\chi_{\mathrm{a} 0}, D_{0}\right)
$$

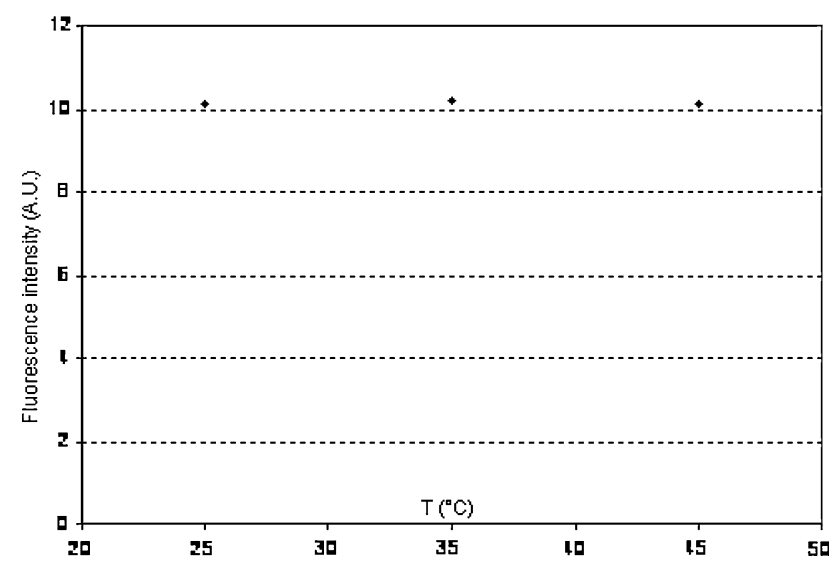

Fig. 13 Fluorescence intensity as a function of the fuel temperature $\left(Z_{a}=20 \%\right)$

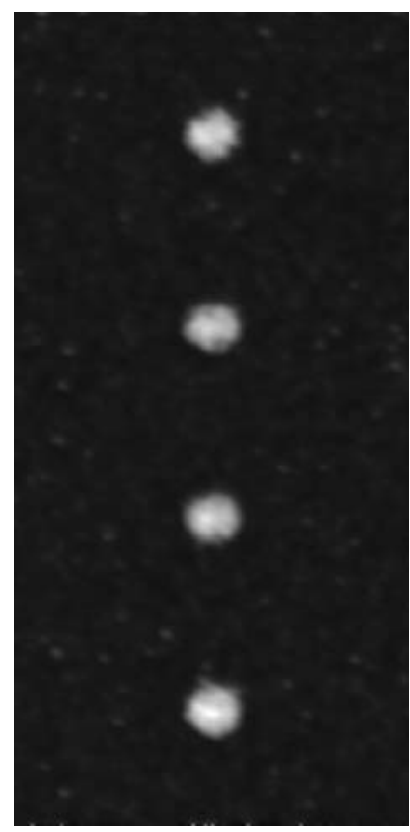

Fig. 14 Illustration of the region of interest

where $I^{*}$ denotes the fluorescence intensity normalized by the incident laser energy.

The determination of the acetone fraction required the inversion of Eq. 13. This inversion is considerably eased by working with a 2D-interpolation of $\zeta$ instead of running the heavy ray tracing code for any guest value of $\chi_{\mathrm{a}}$. The interpolated function is obtained from a relatively dense meshing of the space $\left(\chi_{\mathrm{a}}, D\right)$.

\subsection{Preliminary test}

The technique was preliminarily tested on droplets injected at ambient temperature, for which no significant size reduction can be noticed. Measurements of fluorescence intensity are presented in Fig. 15 for several positions within the droplet stream. The initial conditions were $Z_{\mathrm{a} 0}=20 \%$ and $D=106 \mu \mathrm{m}$. The fluorescence intensity remains within $\pm 1 \%$ RMS, which provides an estimate of the accuracy of the measurement of the fluorescence intensity.

\subsection{Experimental results for combusting binary droplets}

Droplets with a diameter on the order of $100 \mu \mathrm{m}$ were also considered in this study. Figure 16 depicts the variation of the fluorescence intensity with the distance from the nozzle exit for three initial compositions $Z_{\mathrm{a} 0}=30 \%, Z_{\mathrm{a} 0}=20 \%$ 


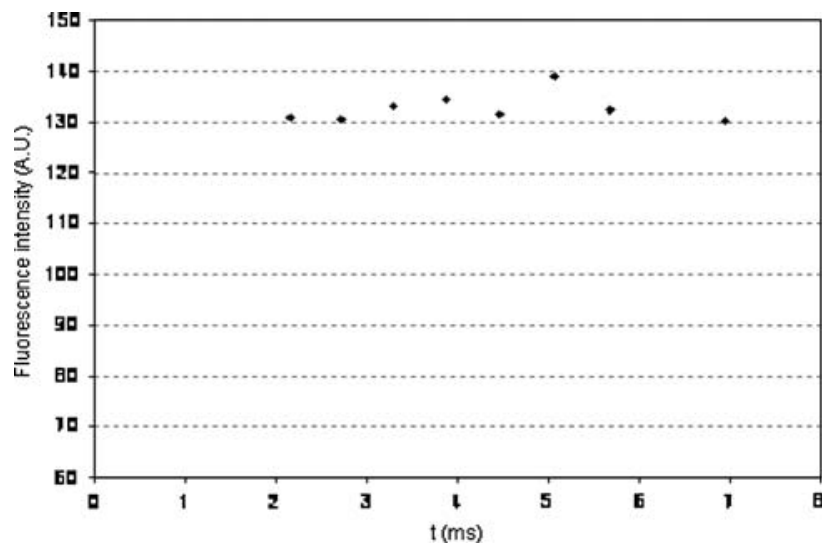

Fig. 15 Test of receptiveness. Records of fluorescence intensities meassured in a monodisperse droplet stream $\left(Z_{a}=20 \%\right.$, $D=106 \mu \mathrm{m})$ at different times from the injection andj under quasinon-evaporating conditions

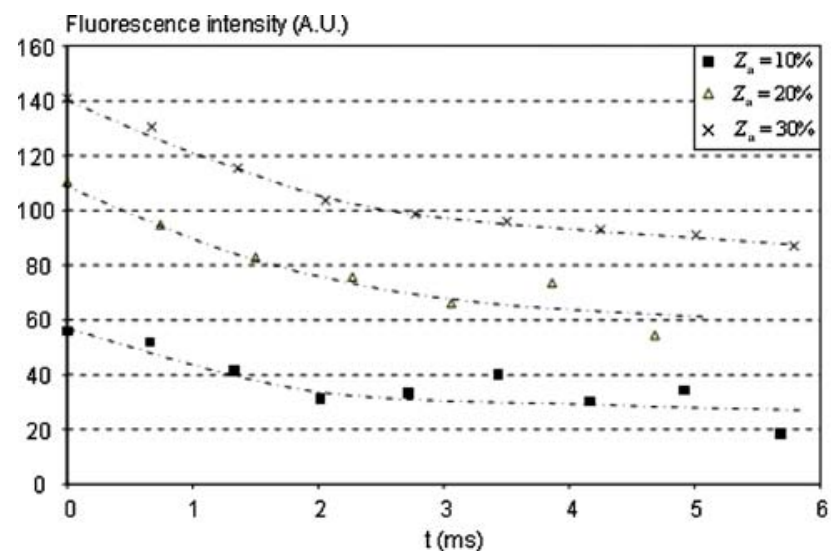

Fig. 16 Temporal evolution of the fluorescence intensity. Case of combusting monodisperse droplets streams $\left(\mathrm{Z}_{\mathrm{a}}=10 \% ; D_{0}=\right.$ $95.3 \mu \mathrm{m}, \mathrm{Z}_{\mathrm{a}}=20 \% ; D_{0}=99.5 \mu \mathrm{m}$ and $\left.\mathrm{Z}_{\mathrm{a}}=30 \% ; D_{0}=97.9 \mu \mathrm{m}\right)$

Table 1 Operating conditions

\begin{tabular}{llll}
\hline$D_{0}(\mu \mathrm{m})$ & 95.3 & 99.5 & 97.9 \\
$Z_{a 0}(\%)$ & 10 & 20 & 30 \\
$C$ & 3.18 & 3.07 & 3.17 \\
$a_{\text {eff }}\left(\mathrm{m}^{-2} / \mathrm{s}\right)$ & $3.9 \times 10^{-9}$ & $4.3 \times 10^{-9}$ & $4.9 \times 10^{-9}$ \\
\hline
\end{tabular}

and $Z_{\mathrm{a} 0}=10 \%$. The operating conditions are summarized in Table 1. The initial diameters differ slightly for the three compositions since it was difficult to achieve perfectly steady droplet streams while maintaining the diameter. The droplet spacing $C$ is also added in Table 1 and is almost identical for the three investigated conditions.

Distance from the injector exit was converted into time by means of the local droplet velocity measured by LDA. The origin time is taken at the exit of the ignition device. As

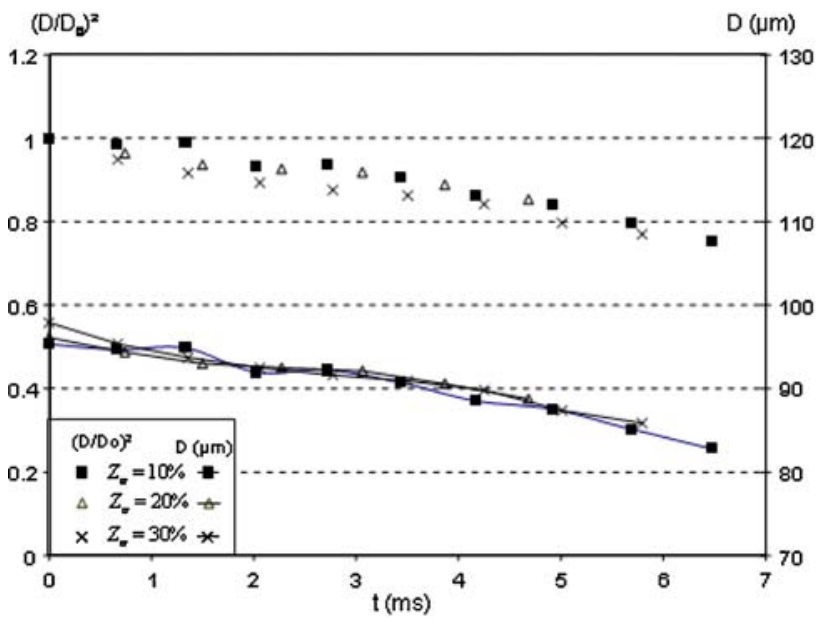

Fig. 17 Temporal evolution of the raw and squared diameters measured for $\mathrm{Z}_{\mathrm{a}}=10 \%, \mathrm{Z}_{\mathrm{a}}=20 \%$ and $\mathrm{Z}_{\mathrm{a}}=30 \%$

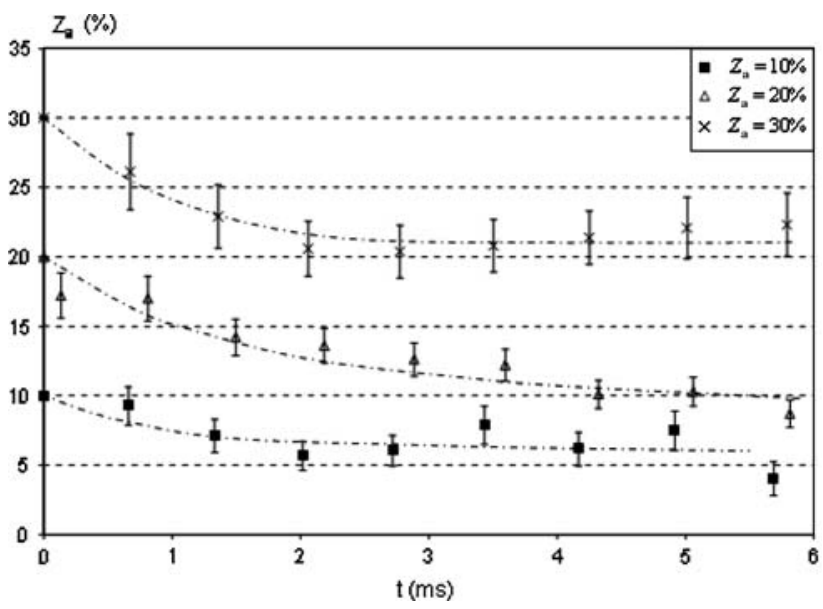

Fig. 18 Measured evolution of the acetone volume fraction as a function of time $\left(\mathrm{Z}_{\mathrm{a}}=10 \%, \mathrm{Z}_{\mathrm{a}}=20 \%\right.$ and $\left.\mathrm{Z}_{\mathrm{a}}=30 \%\right)$

expected, the fluorescence intensity decreases with the time elapsed from the injection. This decrease comes from the preferential depletion of acetone, which is the more volatile component in the mixture. The temporal evolution of the droplet diameter is presented in Fig. 17. The droplet shrinkage seems to follow a $D^{2}$ law (Kuo 2005). A perfect sphericity is required for the size measurement but is difficult to insure shortly after the break-up. For this reason, size measurements do not start exactly at the same distance from the injector exit depending on the tested conditions. The acetone volume fraction can be inferred from the measured fluorescence intensity according to Eq. (13). The temporal evolution of the acetone volume fraction is shown in Fig. 18. The first measurement point at $t=0$ corresponds to the injection conditions. The depletion of acetone is the quickest at the beginning of the evaporation then it slows 
down considerably in a second phase. Initially, the droplets are rather cold and only acetone evaporates significantly. Then, since the droplets are heated up, ethanol begins to participate increasingly to the vaporisation flow. With the rather strong initial vaporization of acetone, ethanol becomes extremely in excess near the surface of the droplet. Even if the saturation pressure of ethanol is much lower than the one of acetone. According to the Raoult's law, at the liquid/vapour interface of an ideal mixture, the molar fraction of the vapour of each component is proportional to their liquid fraction. For this reason, the vaporisation rate of ethanol increases whereas the one of acetone slows down which ultimately results in a stagnation of the space-averaged acetone fraction (phase 2). The whole acetone present in the core of the droplet must diffuse to the surface of the droplet before that the surface temperature can get higher than the boiling temperature of acetone and the vaporization rate of ethanol increases further again. To compare safely the evolutions of the previous droplets streams, the results are put in a dimensionless form. The time is normalized by the acetone diffusion time $\frac{D^{2}}{a_{\text {eff }}}$, where $a_{\text {eff }}$ is the effective diffusivity depending on the droplet Peclet number as defined by Abramzon and Sirignano (1989), whereas the acetone volume fraction is divided by its initial value. The values of $a_{\text {eff }}$ are quoted in Table 1. As expected, the normalized volume fraction of acetone (Fig. 19) seems to follow the same trend regardless to the initial conditions, i.e., the composition and the droplet diameter. The more scattered points are for the case $Z_{\mathrm{a}}=10 \%$, for which the relative uncertainty is the largest.

\subsection{Discussion about the technique accuracy}

The accuracy of the technique can be inferred from Eq. (13), which can be stated as followed:

$f\left(I^{*}, I_{0}^{*}, \mu, \chi_{\mathrm{a} 0}, D, \varepsilon, D_{0}\right)=g\left(\mu, \chi_{\mathrm{a}}, D, \varepsilon\right)$

The uncertainty $\Delta \chi_{\mathrm{a}}$ is then derived from Eq. (14) after having considered the respective sensibilities of $f$ and $g$ to the parameters $\mu, I^{*}, I_{0}^{*}, D, \varepsilon$ and $D_{0}$ :

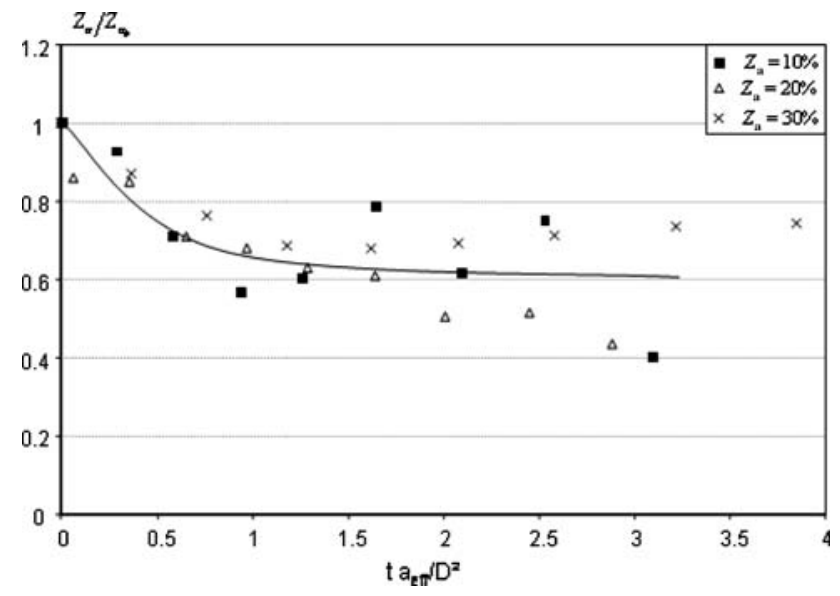

Fig. 19 Evolution of the normalized acetone volume fraction as a function of the dimensionless time for $Z_{a}=10 \%, Z_{a}=20 \%$ and $\mathrm{Z}_{\mathrm{a}}=30 \%$

The differential terms in Eq. (15) are evaluated numerically and the respective influences of each error source are summarized in Table 2. Referring to Section 5.2, it was found that $\left|\Delta I^{*} / I^{*}\right|$ is about $1 \%$, when the droplet size is $106 \mu \mathrm{m}$ and the acetone volume fraction $20 \%$. Assuming a Gaussian process for the statistic convergence of the fluorescence signal, a more general expression can deduced from this case:

$\left|\Delta I^{*} / I^{*}\right|=0.01 \sqrt{\frac{0.2}{Z_{\mathrm{a}}}\left(\frac{106}{D(\mu \mathrm{m})}\right)^{3}}$

Finally, the error bars, calculated according to Eq. (15), after conversion into volume fraction, are reported for each measurement points of Fig. 18. The order of magnitude of the absolute uncertainty $\Delta Z_{\mathrm{a}}$ is respectively $\pm 0.85 \%$ for $Z_{\mathrm{a}_{0}}=10 \%, \pm 1 \%$ for $Z_{\mathrm{a}_{0}}=20 \%$ and $\pm 2.1 \%$ for $Z_{\mathrm{a}_{0}}=30 \%$.

\section{Concluding remarks}

This paper presents one of the first attempts to measure the transient evolution of the composition of binary droplets.

$\Delta \chi_{\mathrm{a}}=\frac{\left|\frac{\partial f}{\partial \mu}-\frac{\partial g}{\partial \mu}\right| \Delta \mu+\left|\frac{\partial f}{\partial \chi_{a 0}}\right| \Delta \chi_{a 0}+\left|\frac{\partial f}{\partial I^{*}}\right| \Delta I^{*}+\left|\frac{\partial f}{\partial I_{0}^{*}}\right| \Delta I_{0}^{*}+\left|\frac{\partial f}{\partial D}-\frac{\partial g}{\partial D}\right| \Delta D+\left|\frac{\partial f}{\partial \varepsilon}-\frac{\partial g}{\partial \varepsilon}\right| \Delta \varepsilon+\left|\frac{\partial f}{\partial D_{0}}\right| \Delta D_{0}}{\left|\frac{\partial g}{\partial \chi_{\mathrm{a}}}\right|}$ 
Table 2 Influences of the different error sources

\begin{tabular}{lllllll}
\hline$\Delta \mu$ & $\Delta \chi_{a 0}$ & $\Delta I^{*} / I^{*}$ (A.U.) & $\Delta I_{0}^{*} / I_{0}^{*}($ A.U. $)$ & $\Delta D(\mu \mathrm{m})$ & $\Delta \varepsilon=106 \mathrm{~mol}^{-1} \cdot \mathrm{m}^{-1}$ & $\Delta D_{0}(\mu \mathrm{m})$ \\
\hline 0.09 & 0.002 & $0.01 \sqrt{\frac{0.2}{Z_{a}}\left(\frac{106}{D(\mu \mathrm{m})}\right)^{3}}$ & $0.01 \sqrt{\frac{0.2}{Z_{a 0}}\left(\frac{106}{D_{0}(\mu \mathrm{m})}\right)^{3}}$ & 0.5 & 106 & 0.1 \\
\hline
\end{tabular}

The composition is based on two independent measurements:

- the direct measurement of the fluorescence signal of acetone which acts also as a volatile fuel in the mixture, and

- an accurate independent measurement of the droplet diameter using the forward scattering interferometry technique.

The fluorescence is induced by an UV laser at $266 \mathrm{~nm}$. The technique is sensitive to the composition variations when the acetone volume fraction is less than $30 \%$. Above this value, the strong absorption of acetone molecules becomes a serious disadvantage. The technique accuracy is of the order of $2 \%$ in term of acetone volume fraction.

To implement the technique, one of the limitations comes from the fact that the volume fraction is inferred directly from the intensities, which implies a strict controlled of the operating conditions.

In future, these experimental data will be used to assess multicomponent evaporation models taking into account the diffusion within the droplets. Theoretical maps of the acetone fraction inside the droplet will be used to analyse the bias induced by such acetone fraction gradients on the measurements.

Acknowledgments This program has been conducted in the framework of the ASTRA program, supported by CNRS and ONERA. The authors wish to thank Loic Mees and Gérard Gréhan for their help in the Generalized Lorentz-Mie Theory calculations.

\section{References}

Abramzon B, Sirignano WA (1989) Droplet vaporization model for spray combustion calculations. Int J Heat Mass Trans 32:16051618

Bazile R, Stepowski D (1995) Measurements of vaporized and liquid fuel concentration fields in a burning spray jet of acetone using planar laser-induced fluorescence. Exp Fluids 20:1-9

Castanet G, Lavieille P, Lebouché M, Lemoine F (2003) Measurement of the temperature distribution within monodisperse combusting droplets in linear streams using two-color laserinduced fluorescence. Exp Fluids 35:563-571

Castanet G, Lebouché M, Lemoine F (2005) Heat and mass transfer characterization in combusting monodisperse droplets in linear stream. Int J Heat Mass Transfer 48:3261-3275

Connon CS, Dimalanta R, Choi C, Dunn-Rankin D (1997) LIF measurements of fuel vapor in an acetone droplet stream. Combust Sci Technol 129:197-216
Domann R, Hardalupas Y (2001) Spatial distribution of fluorescence intensity within large droplets and its dependence on dye concentration. Appl Opt 40:3586:3597

Domann R, Hardalupas Y, Jones AR (2002) Study of the influence of absorption on the spatial distribution of fluorescence intensity within large droplets using Mie theory, geometrical optics and imaging experiments. Meas Sci Technol 13:280291

Frohn A, Roth N (1998) Dynamics of droplets. Sringer, Heidelberg

Glowacki J (1964) Further investigations into fluorescence quenching of the dyes by neutral salts. Acta Phys Pol 15:767-780

Gouesbet G, Gréhan G (2000) Generalized Lorenz-Mie theories, from past to future. Atomization Sprays 10(3-5):277-333

Koenig G, Anders K, Frohn A (1986) A new light scattering technique to measure droplet diameter of periodically generated moving droplets. J Aerosol Sci 17:157-167

Kuo K (2005) Principles of combustion. Wiley, New Jersey

Lavieille P, Lemoine F, Lavergne G, Lebouché M (2001) Evaporating and combusting droplet temperature measurements using twocolor laser-induced fluorescence. Exp fluids 31:45-55

Lavieille P, Lemoine F, Lebouché M (2002) Experimental investigation on interacting low evaporating droplets temperature in linear stream using two colors laser induced fluorescence. Combust Sci Technol 174(4):117-142

Le Gal P, Farrugia N, Greenhalgh DA (1999) Laser sheet dropsizing of dense sprays. Opt Laser Technol 31:75-83

Lemoine F, Antoine Y, Wolff M, Lebouché M (1999) Simultaneous temperature and 2D velocity measurements in a turbulent heated jet using combined laser-induced fluorescence and LDA. Exp Fluids 26:315-323

Lozano A, Yip B, Hanson RK (1992) Acetone: a tracer for concentration measurements in gaseous flows by planar laserinduced fluorescence. Exp Fluids 13:369-376

Majumder OD, Jayakumar RK, Vatsa SK, Kulshreshtha, Mittal JP (1999) Multiphoton ionisation of acetone at $355 \mathrm{~nm}$ : a timeof-flight, mass spectrometry study. Chem Phys Lett 304: 51-59

Maqua C, Castanet G, Doué N, Lavergne G, Lemoine F (2006) Temperature measurements of binary droplets using three color laser-induced fluorescence. Exp Fluids 40:786-797

Massoli P (1998) Rainbow refractometry applied to radially inhomogeneous spheres: the critical case of evaporating droplets. Appl Opt 37:3227-3235

Orain M, Mercier X, Grisch F (2005) PLIF imaging of fuel vapour spatial distribution in an acetone droplet stream, comparison with modelling. Combust Sci Technol 177:249-278

Ritchie BD, Seitzman JM (2001) Quantitative acetone PLIF in twophase flows. AIAA paper 2001-0414

Silverman MA, Dunn-Rankin D (1994) Experimental investigation of a rectilinear droplet stream flame. Combust Sci Technol 100:5773

Thurber MC, Grish F, BJ Kirby; M Votstmeier; RK Hanson (1998) Measurements and modelling of acetone laser-induced fluorescence with implications for temperature-imaging disgnostics. Appl Opt 37(21):4963-4978

Tran T, Kochar Y, Seitzman J (2005) Measurements of liquid acetone fluorescence and phosphorescence for two-phase fuel imaging. AIAA paper $2005-0827$ 
Wilms J, Weigand, B (2006) Mass transfer in evaporating binary mixture droplets, 10th international conference on liquid atomization and spray systems, Kyoto, Japan

Wilms J, N Roth N, Arndt S, Weigand B (2004) Determination of the composition of multicomponent droplets by rainbow refractometry, 12th symposium on application of laser techniques to fluid mechanics, Lisbon, Portugal
Zhao Y, Qiu HH (2004) Measurements of multicomponent microdroplet evaporation by using novel optical techniques, 12th symposium on application of laser techniques to fluid mechanics, Lisbon, Portugal 